\title{
On the Construction of Invariant Measure over the Orthogonal Group on the Hilbert Space by the Method of Cayley Transformation
}

By

\author{
Hiroaki SHIMOMURA*
}

The purpose of this paper is to construct some invariant measures over the infinite dimensional rotation group, analogously to the Haar measure in the finite dimensional case. In this direction there are some results making use of Haar measure on compact groups or Gaussian measure on Hilbert spaces. See, [5], [6], [9] and [10]. But it seems to me that the treatment of the whole group is complicated and difficult. On the other hand, the Cayley transformation in the finite dimensional Euclid space gives a correspondence between the special orthogonal group and the set of skew-symmetric operators, and still may be useful for the infinite dimensional case. Thus, we restrict our consideration to a subgroup which is included in the domain of Cayley transformation. Then the problem is transformed as follows. To the rotationally invariant measure on this subgroup what measure corresponds on a suitable class of infinite dimensional skew-symmetric operators? In order to solve it we first consider the Cayley image of Haar measure in the $n$-dimensional case and second construct a finitely additive measure as the limit of $n \rightarrow \infty$. Lastly we discuss the countably additive extension of so obtained measure. I like to express my thanks to Prof. H. Yoshizawa for the constant encouragement. Also I thank deeply to Prof. Y. Yamasaki and Prof. T. Hirai for their useful suggestions.

$\S 1$. Some properties of Cayley transformation in the finite dimensional case.

Communicated by H. Yoshizawa, January 9, 1974.

* Graduate School, Kyoto University, Kyoto. 
$\S 2$. The Cayley image of Haar measure and its continuity in the finite dimensional case.

$\S 3$. The cylindrical measure on some class of skew-symmetric operators and its countably additive extension.

\section{$\S 1$. Some Properties of Cayley Transformation in the Infinite Dimensional Case}

Let $H$ be a real separable Hilbert space, $O(H)$ be the orthogonal group on it and $I$ be the identity operator. We start from the following subgroup of $O(H) . \quad O_{0}(H)=\{T \in O(H) ; I-T$ is of finite rank . Various topologies are considerable, but here we give two special norms on this group for our later discussion. One is the operator norm and the other is Hilbert-Shmidt norm. Perhaps the lack of completeness is inconvenient, so we extend $O_{0}(H)$ to larger classes $O_{c}(H)$ and $O_{h}(H)$, where $O_{c}(H)=$ $\{T \in O(H) ; I-T$ is a compact operator $\}, O_{h}(H)=\{T \in O(H) ; I-T$ is a Hilbert-Shmidt operator $\}$. The metric $d_{c}$ and $d_{h}$ are defined as follows. $d_{c}(T, S)=\|T-S\|_{o p}$ and $d_{h}(T, S)=\|T-S\|_{H S}$.

Theorem 1.1. $\left(O_{c}(H), d_{c}\right)$ and $\left(O_{h}(H), d_{h}\right)$ are complete separable metric groups and these two metrics are invariant under the action of $O(H)$ from left and right.

The assertion of the theorem is easily checked, so that we omit the proof.

Lemma 1.1. Let $T$ belong to $O_{c}(H)$. If $T$ has not eigen value - 1 , then $(I+T)$ has a bounded inverse.

Proof is omitted. See [11].

The subset of $O_{c}(H)$ appeared in the above lemma will be denoted by $G_{c}$ and $G_{c} \cap O_{h}(H)$ will be denoted by $G_{h}$.

Lemma 1.2. $G_{c}$ and $G_{h}$ are open sets in $\left(O_{c}, d_{c}\right)$ and $\left(O_{h}, d_{h}\right)$ respectively. 
Proof. The natural mapping from $\left(O_{h}, d_{h}\right)$ to $\left(O_{c}, d_{c}\right)$ is trivially continuous and $G_{h}=G_{c} \cap O_{h}$, so that we have only to prove in the case of compact one. Supposing $T \in G_{c}$, we shall prove that if $\|S\|_{o p}<\frac{1}{\pi(I+T)^{-1}} \|_{o p}$, $T+S$ has not eigen value -1 . The relation $(T+S) x=-x$ implies $S x=$ $-(I+T) x$, hence $(I+T)^{-1} S x=-x$, so that we have $1 \leqq\left\|(I+T)^{-1} S\right\|_{o p}$ $\leqq\left\|(I+T)^{-1}\right\|_{o p}\|S\|_{o p}$, which is a contradiction.

Q.E.D.

Let $S_{c}(H), S_{h}(H), S_{n}(H)$ be the sets of skew-symmetric compact, skew-symmetric Hilbert-Shmidt, and skew-symmetric nuclear operators on the Hilbert space $H$, respectively. We equip the operator norm, the Hilbert-Shmidt norm and the trace norm in these spaces, respectively.

Theorem 1.2. The infinite dimensional Cayley transformation $C_{\infty}(U)=(I-U)(I+U)^{-1}$ is an onto homeomorphism from $G_{c}$ and $G_{h}$ to $S_{c}(H)$ and $S_{h}(H)$ respectively and the inverse mapping has the same form as $C_{\infty}$.

Proof. The algebraic assertion and onto properties are the same as for the finite dimensional case, so that we only check the continuity property. Before the proof we state several lemmas.

Lemma 1.3. Let $X$ be a bounded operator. If $\|X\|_{o p}<1,(I-X)$ has a bounded inverse and $\left\|I-(I-X)^{-1}\right\|_{o p} \leqq \frac{\|X\|_{o p}}{1-\|X\|_{o p}}$.

Lemma 1.4. Let $X$ be a bounded operator. If $X$ has a bounded inverse, the mapping $X \rightarrow X^{-1}$ is continuous with respect to the operator norm.

The above two lemmas are well known. See [12].

Proof of theorem 1.2. Suppose $U_{n} \rightarrow U_{0}$ in $G_{\alpha}$, where $\|\cdot\|_{\alpha}$ is the operator norm or Hilbert-Shmidt norm. Then we have

$$
\begin{aligned}
& \left\|\left(I+U_{n}\right)^{-1}\left(I-U_{n}\right)-\left(I+U_{0}\right)^{-1}\left(I-U_{0}\right)\right\|_{\alpha} \\
\leqq & \left\|\left(I+U_{n}\right)^{-1}\left(I-U_{n}\right)-\left(I+U_{n}\right)^{-1}\left(I-U_{0}\right)\right\|_{\alpha} \\
+ & \left\|\left(I+U_{n}\right)^{-1}\left(I-U_{0}\right)-\left(I+U_{0}\right)^{-1}\left(I-U_{0}\right)\right\|_{\alpha} \\
\leqq & \left\|\left(I+U_{n}\right)^{-1}\right\|_{o p}\left\|U_{n}-U_{0}\right\|_{\alpha}+\left\|\left(I+U_{n}\right)^{-1}-\left(I+U_{0}\right)^{-1}\right\|_{o p}\left\|I-U_{0}\right\|_{\alpha}
\end{aligned}
$$


Thus the assertion will be proved by the above inequality together with Lemma 1.4. The same argument holds for the inverse mapping. Q.E.D.

Remark. (1) The set $G_{\alpha}$ (where $a$ is $c$ or $h$ ) is invariant under the operation of group inverse and the action of $T(\cdot) T^{-1}$ for all $T \in O(H)$.

(2) $C_{\infty}(I)=0, \quad C_{\infty}\left(U^{-1}\right)=-C_{\infty}(U)$ and $C_{\infty}\left(T U T^{-1}\right)=T C_{\infty}(U) T^{-1}$ for all $T \in O(H), U \equiv G_{\alpha}$.

\section{$\S 2$. The Cayley Image of Haar Measure and Its Continuity in the Finite Dimensional Case.}

Let $S O(n)$ be the special orthogonal group on the $n$-dimensional Euclid space, $\mu_{n}$ be the normalized Haar measure on it and $C_{n}$ be the Cayley transformation from $S O(n)$ to $S(n)$, where $S(n)$ is the set of $n$ dimensional skew-symmetric matrices. The domain of $C_{n}$ is the set of operators which have not eigen value -1 and will be denoted by $G_{n}$.

Lemma 2.1. $\mu_{n}\left(G_{n}\right)=1$ and $G_{n}$ is a open dense set in $S O(n)$ in the natural topology.

The assertion is easily checked, so that we omit it.

Naturally $S(n)$ may be considerable as $\frac{1}{2} n(n-1)$-dimensional Euclid space, regarding the entries of the upper part of the diagonal as independent variables. Symbolically we shall write $d A=d a_{1,2} d a_{1,3} \ldots d a_{n-1, n}$ for the volume element of the Lebesgue measure in this space.

Theorem 2.1. Let $\nu_{n}$ be the image of $\mu_{n}$ under the map $C_{n}$. Then it has the following explicit form. $\nu_{n}(E)=\gamma_{n} \int_{E} \frac{d A}{\operatorname{det}(I+A)^{n-1}}$; where $\gamma_{n}$ is a normalizing constant, and $E$ is a Borel set.

Proof. If $X, X_{1}$, and $X_{2}$ are sufficiently near to 0 , then

$$
\begin{aligned}
& (I-X)(I+X)^{-1}=I-2 X+2 X^{2}-\ldots, \quad \text { and } \\
& \left(I-X_{1}\right)\left(I+X_{1}\right)^{-1}\left(I-X_{2}\right)\left(I+X_{2}\right)^{-1}=I-2\left(X_{1}+X_{2}\right)+\ldots
\end{aligned}
$$

Thus, an infinitesimal rotation on $S O(n)$ corresponds to an infinitesimal 
translation on $S(n)$, therefore the invariance property of $\mu_{n}$ implies that $\nu_{n}$ is approximately Lebesgue at origin up to a constant factor; $d \nu_{n}=\gamma_{n}$ $d X$ as denoted symbolically. Let $A_{0}=\left(I-U_{0}\right)\left(I+U_{0}\right)^{-1}$ be an arbitrary element in $S(n)$, where $U_{0} \in S O(n)$. The transformation $W(X)$ $=C_{n}\left(C_{n}^{-1}(X) \cdot U_{0}\right)$ is a measure preserving map on $S(n)$ and $W(0)=\mathrm{A}_{0}$, so that $d \nu_{n}(0)=d\left(W \nu_{n}\right)(0)=d \nu_{n}\left(A_{0}\right)$. If we neglect more than first order terms, then $A=W(X) \sim A_{0}+\left(I+A_{0}\right) X\left(I-A_{0}\right)$ and $d A=\frac{\partial(A)}{\partial(X)} d X=$ $\frac{1}{\gamma_{n}} \frac{\partial(A)}{\partial(X)} d \nu_{n}\left(A_{0}\right)$, where $\frac{\partial(A)}{\partial(X)}=\frac{\partial\left(a_{1,2} a_{1,3} \ldots a_{n-1, n}\right)}{\partial\left(x_{1,2} x_{1,3} \ldots x_{n-1, n}\right)}$ is a Jacobian. In order to complete the proof, we have only to check the following lemma.

Lemma 2.2. If $A=\left(A_{0}+I\right) X\left(I-A_{0}\right)$, then $\frac{\partial(A)}{\partial(X)}=\operatorname{det}\left(I+A_{0}\right)^{n-1}$, where all matrices are elements of $S(n)$.

This is an elementary calculation and can be proved by induction for n. We omit it.

Remark. (1) For the case $n=2, \nu_{2}$ is one dimensional Cauchy distribution and its Fourier transformation becomes $\exp (-|x|)$.

(2) $\nu_{n}\left(T E T^{-1}\right)=\nu_{n}(E)$ for all $T \in O(n)$ and Borel sets.

We put $\langle A, B\rangle_{n}=\frac{1}{2} \operatorname{tr}(B * A)=-\frac{1}{2} \operatorname{tr}(B A)$ for $A, B \in S(n)$. It is a scalar product on $S(n)$, and $\|A\|_{n}^{2}=\langle A, A\rangle_{n}$ is the Hilbert-Shmidt norm.

We define a linear mapping $\pi_{n, m}$ from $S(n)$ to $S(m)(n \geqq m)$.

$$
\pi_{n, m}:\left(\begin{array}{c|c}
A_{m} & * \\
-* & * *
\end{array}\right) \longrightarrow A_{m}
$$

Theorem 2.2. The sequence $\left\{\nu_{n}\right\}$ is consistent relative to the maps $\pi_{n, m}$ and the normalizing constant $\gamma_{n}$ can be calculated by the following recurrent formula.

$$
\frac{\gamma_{n}}{\gamma_{n \vdash 1}}=\int_{R^{n}} \frac{d y_{1}}{\left(1+\| y_{2} \ldots d y_{n}\right.}
$$

Proof. Let $\chi_{n}(X)$ be the Fourier transformation of $\nu_{n}$, namely 
$\chi_{n}(X)=\int_{S(n)} e^{i\langle A, X\rangle_{n}} d \nu_{n}(A)$. If we write $A=\left(\begin{array}{cc}\mathrm{A}_{1} & a \\ -t_{a} & 0\end{array}\right), \quad X=\left(\begin{array}{cc}X_{1} & x \\ -{ }^{t} x & 0\end{array}\right)$, where $A_{1}, X_{1}$ are $(n-1)$-dimensional matrices and $a, x$ are $(n-1)$-dimensional vectors, then, since $\operatorname{det}(I+A)=\operatorname{det}\left(I+A_{1}\right)\left\{1+\left\langle\left(I-A_{1}^{2}\right)^{-1} a, a\right\rangle\right\}$, we have

$$
\begin{aligned}
\chi_{n}(X) & =\gamma_{n} \int \frac{e^{i\left\langle A_{1}, X_{1}\right\rangle_{n-1} e^{i\langle a, x\rangle} d a d A_{1}}}{\operatorname{det}\left(I+A_{1}\right)^{n-1}\left\{1+\left\langle\left(I-A_{1}^{2}\right)^{-1} a, a\right\rangle\right\}^{n-1}} \\
& =\gamma_{n} \int \frac{e^{i\left\langle A_{1}, X_{1}\right\rangle_{n-1} e^{i\left\|\left(I+A_{1}\right) x\right\| y_{1}}}}{\operatorname{det}\left(I+A_{1}\right)^{n-2}\left(1+\|y\|^{2}\right)^{n-1}} d A_{1} d y_{1} \ldots d y_{n-1}
\end{aligned}
$$

If $x=0$, then $\chi_{n}(X)=\chi_{n-1}\left(X_{1}\right)$ with the requested result for $\frac{\gamma_{n}}{\gamma_{n-1}}$. In terms of the measure this shows $\pi_{n, n-1} \nu_{n}=\nu_{n-1}$. On the other hand, $\pi_{n, k}=\pi_{m, k} \pi_{n, m}(k \leqq m \leqq n)$ holds trivially. Combining these facts, the assertion is proved.

Q.E.D.

Lemma 2.3. In the notation of Theorem 2,2, $\left|\chi_{n}(X)-\chi_{n-1}\left(X_{1}\right)\right|$ $\leqq\|x\|$.

Proof. Put $X^{\prime}=\left(\begin{array}{cc}X_{1}, & -x \\ t_{x}, & 0\end{array}\right)$, then we have $\chi_{n}(X)=\chi_{n}\left(X^{\prime}\right)$, because $\operatorname{det}(I+A)$ is a even function of $a$. Therefore we have

$$
\begin{aligned}
\chi_{n-1}\left(X_{1}\right)-\chi_{n}(X) & =\chi_{n-1}\left(X_{1}\right)-\frac{1}{2}\left(\chi_{n}(X)+\chi_{n}\left(X^{\prime}\right)\right) \\
& =\gamma_{n} \int \frac{e^{i\left\langle A_{1}, X_{1}\right\rangle_{n-1}(1-\cos \langle a, x\rangle)}}{\operatorname{det}(I+A)^{n-1}} d A,
\end{aligned}
$$

so that

$$
\left|\chi_{n-1}\left(X_{1}\right)-\chi_{n}(X)\right| \leqq\left|1-\chi_{n}(\hat{X})\right|, \text { where } \hat{X}=\left(\begin{array}{cc}
0 & x \\
-t_{x} & 0
\end{array}\right) .
$$

Since $\chi_{n}(\hat{X})=\chi_{n}\left(T \hat{X} T^{-1}\right)$ for all $T$ in $S O(n)$ and $T \hat{X} T^{-1}=\left(\begin{array}{ccc}0 & \|x\| & 0 \\ -\|x\| & 0 & \\ 0 & & \ddots\end{array}\right)$ for some $T \in S O(n)$, by the remark after Lemma $2.2 \chi_{n}(\hat{X})=\exp (-\|x\|)$, and $\left|\chi_{n-1}\left(X_{1}\right)-\chi_{n}(X)\right| \leqq 1-\exp (-\|x\|) \leqq\|x\|$.

Theorem 2.3. Again we use the notation of Theorem 2.2. Then, 
$\left|1-\chi_{n}(X)\right| \leqq \frac{1}{2}\|X\|_{\text {tr }}$, where $\|\cdot\|_{\text {tr }}$ is the trace norm.

Proof. By the remark (2) after Lemma 2.2, we can assume $X$ to be in the canonical form without loss of generality.

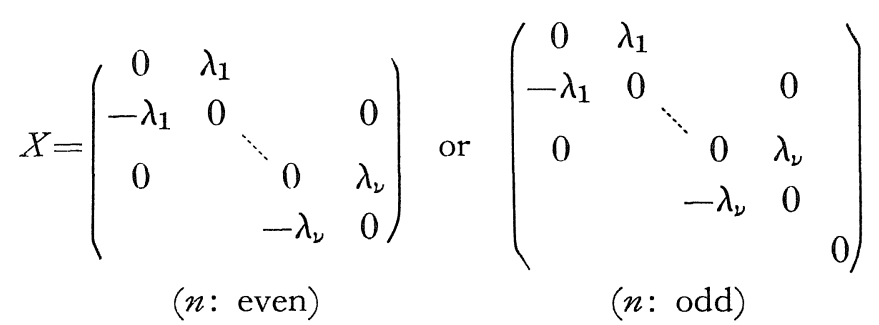

Making use of Lemma 2.3 repeatedly, in both cases we get the following inequality. $\left|1-\chi_{n}(X)\right| \leqq \sum_{j=1}\left|\lambda_{j}\right|=\frac{1}{2}\|X\|_{\text {tr. }}$.

Q.E.D.

We remark that the above estimate does not depend on the dimension.

\section{$\S 3$. The Cylindrical Measure on Some Class of Skew-Sym- metric Operators and Its Countably Additive Extension}

In this section we shall denote the collection of finite dimensional linear subspaces of $H$ by $L^{f}$. If $M$ is the element of $L^{f}, S O(M), S(M), \mu_{M}$, $\nu_{M}$ and $\chi_{M}$ have the same meaning as the the preceding section. We define the mappings $\pi_{N, M}$ from $S(N)$ to $S(M)$ as follows $(N \supseteq M) ; \pi_{N, M}(A)=$ $P_{N, M} A \mid M$, where $P_{N, M}$ is the projection from $N$ to $M$ and $\cdot \mid M$ means the restriction to $M$. Then the discussion of section 2 tells us that $\left\{\nu_{M}: M \in\right.$ $\left.L^{f}\right\}$ is a consistent sequence relative to the maps $\pi_{N, M}$. In order to obtain $\sigma$-additive extension of $\left\{\nu_{M}\right\}$ we shall briefly discuss the projective limit method. First of all we take a sequence of operators $\left\{A_{M}\right\}$ which has the following two properties.
(1) $A_{M} \in S(M)$.
(2) $\pi_{N, M}\left(A_{N}\right)=A_{M} \quad(N \supseteq M)$.

Then we can define a bilinear functional $B$ on $H \times H$ such that $B(x, y)$ $=\left\langle A_{M} x, y\right\rangle$, where $M$ includes both $x$ and $y$. It is well defined independent from the choice of $M$ by the property (2) and satisfies $B(x, y)$ $=-B(y, x)$. Conversely if a bilinear skew-symmetric functional $B$ is 
given, $B(x, y)=\left\langle\mathrm{A}_{M} x, y\right\rangle x, y \in M$ determines the operator $A_{M} \in S(M)$ and the sequence $\left\{A_{M}\right\}$ satisfies (2). Evidently this correspondence is one to one. Thus,

Theorem 3.1. The projective limit space of $\left\{S(M), \pi_{N, M}\right\}$ is realized as the set of bilinear skew-symmetric functionals on $H \times H$.

Theorem 3.2. A $\sigma$-additive extension of $\left\{\nu_{M}\right\}$ exists uniquely on the projective limit space of $\left\{S(M), \pi_{N, M}\right\}$.

The proof is carried out by Bochner's famous theorem. We omit it.

Though it can be shown more directly, Theorem 3.2 tells us that we can define a positive definite function $\chi$ on the set $S_{0}(H)$ of all finite rank skew-symmetric operators on $H$.

More exactly, $\chi$ has the following two properties.

(1) $\chi$ is a positive definite function and $\chi(0)=1$.

(2) $\chi(A)=\chi_{M}(A)$ for any $M \in L^{f}$ such that $A H \subseteq M$.

The last theorem in Section 2, together with the fact that $S_{0}(H)$ is dense in $S_{n}(H)$ enables us to extend $\chi$ to $S_{n}(H)$ uniquely. To say repeatedly, $S_{n}(H)$ is the set composed of the skew-symmetric trace class operators which is equipped with the trace norm.

Theorem 3.3. The dual of the space of compact linear operators on $H$ equipped with the operator norm is identified with the space of nuclear operators on $H$ equipped with the trace norm. More exactly, for the dual element $F$, there exists one and only one nuclear operator $T$, such that $F(A)=\operatorname{tr}\left(T^{*} A\right)$ and $\|F\|=\|T\|_{\text {tr. }}$

This is a well known result. We omit it. See [7].

Collorary. The dual of $S_{c}(H)$ is identified with $S_{n}(H)$ in the above sense.

Proof. Using Hahn-Banach theorem, we can easily reduce it to the above theorem, so that we omit it. 
Theorem 3.4. A unique cylindrical measure (weak distribution) exists on $S_{c}(H)$ such that its finite dimensional projection is given by the Cayley image of Haar measure.

Proof. Since a weak distribution corresponds uniquely to a continuous positive definite functional on its dual space, we have only to check the continuity of $\chi$. But $\left|1-\chi\left(X^{\prime}\right)\right| \leqq \frac{1}{2}\|X\|_{\text {tr }}$ always holds, so that we complete the proof. In details, see [3].

(1) Consider the spectral decomposition of $T \in S_{n}(H)$

$$
\begin{aligned}
T(X) & =\sum \lambda_{j}\left\{\left\langle x, e_{2 j}\right\rangle e_{2 j-1}-\left\langle x, e_{2 j-1}\right\rangle e_{2 j}\right\} . \text { Then } \\
\chi(T) & =\int_{S_{c}(H)} \exp \left\{i \frac{1}{2} \operatorname{tr}\left(T^{*} A\right)\right\} d \nu(A) \\
& =\lim _{n} \gamma_{2 n} \int_{S(2 n)} \frac{\exp \left\{i \sum_{j=1}^{n} \lambda_{j} a_{2 j-1,2 j}\right\}}{\operatorname{det}(I+A)^{2 n-1}} d A
\end{aligned}
$$

(2) Let $e$ and $f$ be the elements in $H$. The function $\xi_{e, f}(A)=\langle A e, f\rangle$ is of course measurable and its distribution is Cauchy. For real $y$,



(3) $\nu(E)=\nu(-E)$ and $\nu\left(T E T^{-1}\right)=\nu(E)$ for all $T \in O(H)$ and measurable set $E$.

Our final problem in this paper is the $\sigma$-additive extension of this weak distribution. Apparently its characteristic functional tells us the lack of $\sigma$-additiveness on $S_{c}(H)$. So that we must extend $S_{c}(H)$ to a larger space which is so called a nuclear extension. As the projective limit in Theorem 3.1 is considered to be the largest extension, we have only to check the support of the measure in Theorem 3.2. But on the other hand, since we wish to obtain a measure on $O(H)$, the extension on $S_{c}(H)$ is desirable. Then the problem is set up as follows. Let $T_{1}$ and $T_{2}$ bc bounded operators on $H$ and consider the map, $A \rightarrow T_{1} A T_{2}$, where $A$ belong to $S_{c}(H)$. Then whether the image of $\nu$ by this transformation has 
a $\sigma$-extension on $S_{c}(H)$ or not? Before answering it, we prove the following lemma. ${ }^{1)}$

Lemma 3.1. Let $T_{1}$ and $T_{2}$ be bounded operators on $H$ and assume that $T_{1} A T_{2} \in S_{c}(H)$ for all $A \in S_{c}(H)$. Then, (a) $T_{1}^{*}=c T_{2}$ for some real constant, or (b) $T_{1} A T_{2}=0$ for all $A \in S_{c}(H)$.

Proof. If the rank of $T_{2}$ is less than 1 , then the rank of $T_{1} A T_{2}$ is also less than 1 , but such a skew-symmetric operator must be zero. This is the case (b).

The skew-symmetricity of $T_{1} A T_{2}$ implies that for any $A \in S_{c}(H)$ and $x \in H$, we have $\left\langle A T_{2} x, T_{1}^{*} x\right\rangle=0$. This means that for some real number $C_{x}, T_{1}^{*} x=C_{x} T_{2} x$ if $T_{2} x \neq 0$. Thus, we have only to prove (1) $C_{x}=C_{y}$ for $x \neq y$, and (2) $T_{2} x=0$ implies $T_{1}^{*} x=0$. First we shall prove (2). If $T_{2} x=0$ and $T_{2} y \neq 0$, we have $T_{1}^{*} x=T_{1}^{*}(x+y)-T_{1}^{*} y=$ $\left(C_{x+y}-C_{y}\right) T_{2} y$. Thus, $T_{1}^{*} x$ is linearly dependent with $T_{2} y$. If the rank of $T_{2}$ is more than 2 , this means $T_{1}^{*} x=0$. Next we shall prove (1). If $T_{2} x \neq 0$ and $T_{2} y \neq 0$, we have $T_{1}^{*}(x+y)=T_{1}^{*} x+T_{1}^{*} y=C_{x} T_{2} x+C_{y} T_{2} y=$ $C_{x+y} T_{2}(x+y)=C_{x+y} T_{2} x+C_{x+y} T_{2} y$. If $T_{2} x$ and $T_{2} y$ are linearly independent, comparing the coefficients we get $C_{x+y}=C_{x}=C_{y}$. If $T_{2} x$ and $T_{2} y$ are linearly dependent, $T_{2} x=\alpha T_{2} y$ for some $\alpha$. Then, in virtue of (2) we have $T_{1}^{*} x=\alpha T_{1}^{*} y$, namely $C_{x} T_{2} x=a C_{y} T_{2} y=C_{y} T_{2} x$, hence $C_{x}=C_{y}$.

Q.E.D.

Theorem 3.5. Let $T$ be a bounded operator on $H$ and put $\tau(A)=$ $T^{*} A T$. Then $\tau$ is a bounded transformation on $S_{c}(H)$. In order that $\tau \nu(E)=\nu\left(\tau^{-1}(E)\right)$ has a $\sigma$-additive extension on $S_{c}(H)$, it is sufficient that $T$ is a nuclear operator. Further under the above condition the support of $\tau \nu$ is always contained in $S_{h}(H)$.

Proof. We assume that $T$ is a nuclear operator. Then the range of $\tau$ is included in $\mathrm{S}_{h}(H)$. Thus, it will be sufficient to show that $\tau \nu$ has a $\sigma$-additive extension on $S_{c}(H)$. We shall observe its Fourier transformation: $\widehat{\tau \nu}(B)=\int_{X \in S_{h}(H)} e^{i \operatorname{tr}\left(X^{*} B\right)} d \tau \nu(X)$, where $B$ is an element in $S_{h}(H)$.

1) This lemma was improved by Y. Yamasaki. 
Then by the continuity of $\nu$

$1-\widehat{\tau \nu}(B)=\int_{S c(H)} 1-e^{-i \operatorname{tr}\left(T^{*} A T B\right)} d \nu(A)=\operatorname{Sup}_{\|A\| \leq 1}\left|\operatorname{tr}\left(T^{*} A T B\right)\right|=\left\|T B T^{*}\right\|_{\text {tr }}$.

We put $P_{T}(B)=\operatorname{Sup}_{\|A\| \leq 1}\left|\operatorname{tr}\left(T^{*} A T B\right)\right|$, then it is a semi norm. Naturally the space $S_{h}(H)$ becomes a Hilbert space with the Hilbert-Schmidt norm: $\left\langle B_{1}, B_{2}\right\rangle=\frac{1}{2} \sum_{j=1}^{\infty}\left\langle B_{1} e_{j}, B_{2} e_{j}\right\rangle$, where $e_{1}, e_{2}, \ldots, e_{n}, \ldots$ is a complete orthonormal system (c.o.n.s) in $H$. Put $q_{i, j}(x)=\left\langle x, e_{i}\right\rangle e_{j}-\left\langle x, e_{j}\right\rangle e_{i}$, then the sequence of operators $\left\{q_{i, j}\right\} \in S_{h}(H): i, j=12 \ldots, i<j$ forms a c.o.n.s. in $S_{h}(H)$. After some caluculations we can show that $P_{T}\left(q_{i, j}\right)=2\left\|T e_{i}\right\|\left\|T e_{j}\right\|$, therefore we have $\sum_{i<j} P_{T}\left(q_{i, j}\right)=\sum_{i<j} 2\left\|T e_{i}\right\|\left\|T e_{j}\right\|<\infty$. We define an operator $W$ on $S_{h}(H)$ as follows : $W(B)=\sum_{i<j}\left\langle B, q_{i, j}\right\rangle P_{T}\left(q_{i, j}\right) q_{i, j}$. Then $W$ is a trace class operator on $S_{h}(H)$ and $P_{T}(B) \leqq \sum_{i<j}\left|\left\langle B, q_{i, j}\right\rangle\right| P_{T}\left(q_{i, j}\right)$ $\leqq\left(\sum_{i<j}\left\langle B, q_{i, j}\right\rangle^{2} P_{T}\left(q_{i, j}\right)\right)^{\frac{1}{2}}\left(\sum_{i<j} P_{T}\left(q_{i, j}\right)\right)^{\frac{1}{2}} \leqq\langle W(B), B\rangle^{\frac{1}{2}}\|W\|_{\mathrm{tr}}^{\frac{1}{2}} \cdot \quad$ Therefore $\widehat{\tau \nu}$ is continuous with respect to the norm $\|B\|_{W}=\langle W(B), B\rangle^{\frac{1}{2}}$, and since $W$ is trace class, $\tau \nu$ is a $\sigma$-additive measure on $S_{h}(H)$. See [4]. Q.E.D.

Theorem 3.6. We use the notation of Theorem 3.5. In order that $\tau \nu$ has a $\sigma$-additive extension on $S_{B}(H)$, it is necessary that $T$ is a Hilbert-Shmidt operator, where $S_{B}(H)$ is the set of all bounded skewsymmetric operators.

Proof. Suppose that $\tau \nu$ has a $\sigma$-additive extension on $S_{B}(H)$. If $T$ vanishes identically, the assertion is trivial, so that we take an element $e$ such that $T e \neq 0,\|e\|=1$. Extending it in a suitable way, we form a c.o.n.s $e_{1}, e_{2}, \ldots, e_{n}, \ldots$ such that $e_{1}=e$. The mapping $\xi$ which sends $A \in S_{B}(H)$ to $A e \in H$ is clearly a measurable mapping. Again we observe a Fourier transformation of a measure $\eta$, which is the image of $\tau \nu$ by the map $\xi$. As $\eta$ is a $\sigma$-additive measure on $H$, for any positive $\varepsilon$, there exist $\delta>0$ and a Hilbert-Schmidt operator $S$ on $H$ such that $|1-\hat{\eta}(y)|<\varepsilon$ for all $\|S y\| \leqq \delta$. However, since

$$
\hat{\eta}(y)=\int_{S_{c}(H)} e^{i\langle T y, A T e\rangle} d \nu(A)=\exp \left(-\sqrt{\|\overline{T e}\|^{2}\|T y\|^{2}}-\overline{\langle T e, T y\rangle^{2}}\right),
$$

the inequality $|1-\hat{\eta}(y)|<\varepsilon$ is equivalent with $\|T e\|^{2}\|T y\|^{2}-\langle T e, T y\rangle^{2}$ 
$<(\log (1-\varepsilon))^{2}$. Next we take an arbitrary element $y$ from $H$. If $\lambda$ is small enough to satisfy $\|\lambda S y\| \leqq \delta$, then $\|T e\|^{2}\|T y\|^{2}-\langle T e, T y\rangle^{2} \leqq \frac{1}{\delta^{2}}$ $(\log (1-\varepsilon))^{2}$. Letting $\frac{1}{\lambda}$ to infimum under the above condition, we get $\|T e\|^{2}\|T y\|^{2}-\langle T e, T y\rangle^{2} \leqq \frac{1}{\delta^{2}}(\log (1-\varepsilon))^{2}\|S y\|^{2}$. Substituting $e_{j}$ for $y$ and summing up over $j$, we have $\|T e\|^{2} \sum_{j}\left\|T e_{j}\right\|^{2}-\left\|T^{*} T e\right\|^{2} \leqq \frac{1}{\delta^{2}}$ $(\log (1-\varepsilon))^{2}\|S\|_{H S}^{2}$. Therefore $\sum_{j}\left\|T e_{j}\right\|^{2}<\infty$, namely $T$ is a HilbertSchmidt operator.

Q.E.D.

Making use of the infinite dimensional Cayley inverse transformation, we can obtain some invariant measures on the group $O_{c}(H)$. The author wishes to discuss various properties of these measures in the subsequent paper.

\section{References}

[1] Gelfand. I. M. and Vilenkin, N. Ya., Generalized functions, Vol. 4, (English trans., Academic Press), 1961.

[2] Parthasarathy, K. R., Probability measure on metric spaces, Academic Press, 1967.

[3] Prohorov, Yu. V., The method of characteristic functionals, Proceedings of the Fourth Berkley Symposium on Mathematical Statistics and Probability, 2, Berkley and Los Angeles, University of California Press, 1961, 403-419.

[4] Sato, H., Gaussian measure on a Banach space and abstract Wiener measure, Nagoya Math. J., 36, (1969), 65-81.

[5] Shale, D., Invariant integration over the infinite dimensional orthogonal group and related spaces, Trans. Amer. Math. Soc. 124, (1966).

[6] Shimomura, H., On the Haar measures over the infinite dimensional space, (to appear).

[7] Treve, F., Topological vector spaces, Distributions and Kernels, Academic Press, 1967.

[8] Umemura, Y., Measures on infinite dimensional vector spaces, Publ. RIMS, Kyoto Univ. 1, (1965), 1-47.

[9] Yamasaki, Y., Invariant measures of the infinite dimensional rotation group, Publ. RIMS, Kyoto Univ. 8, (1972), 131-140.

[10] Yamasaki, Y., Projective limit of Haar measures on $\mathrm{O}(\mathrm{n})$, Publ. RIMS, Kyoto Univ. 8, (1972), 141-150.

[11] Yoshida, K., Functional Analysis 1, Iwanami, 1940, (in Japanese).

[12] Loomis, L. H., An Introduction to Abstract Harmonic Analysis, Van Nostrand, 1953. 